\title{
CrystEngComm
}

\section{Hydration studies of a simple molecular solid}

\author{
Maurice Oduor Okoth, $\uparrow$ Ranko Mirko Vrcelj,,$*$ David Bernard Sheen and John Neil Sherwood
}

\author{
Received 30th September 2011, Accepted 9th December 2011 \\ DOI: 10.1039/c2ce06301h
}

\begin{abstract}
This paper presents novel information on the thermal dehydration and rehydration of the molecular solid, Oxalic Acid Dihydrate. Although the procedure of the overall decomposition process is welldefined, the structural basis and mechanism of the dehydration process has been poorly studied. We show that the dehydration occurs in a planar manner, with a resultant semi-topotactic relationship between hydrated and dehydrated structures, reflected in the molecular packing. During rehydration, the reconstruction of the phase can be seen to occur at a 3-dimensional phase boundary reaction front, i.e. a recrystallisation of the dihydrate on the surface of the reactant product, with the topotactic relationship leading to texturing and possibly epitaxial relationships between partially dehydrated and rehydrated structures. The proposed mechanism is shown to be consistent with the measured kinetics of the process.
\end{abstract}

\section{Introduction}

In recent years, much emphasis has been placed on the understanding of the structure/property relationships of molecular solids, especially regarding their stability, physico-chemical properties and in particular the role and occurrence of polymorphism. One subject which has attracted relatively little interest is that of the stability and reactivity of solvates and particularly, hydrates. The size and importance of this last group of materials is well demonstrated by the facts that the European Pharmacopoeia lists approximately $30 \%$ of its entries as either forming hydrates or associates with water. Also that interrogation of the latest version of the Cambridge Crystallographic Database $^{1}$ (using version 5.31 of ConQuest) returns $\sim 10 \%$ of the available entries with the simple text search for the word "hydrate". Studies of the relative appearance of hydrates using such databases and related computational studies are now relatively common. ${ }^{2}$

Given that so many molecular materials form hydrates (an important subset of general solvates), remarkably little information is available on the behaviour of these systems, even for simple structures. In particular, knowing that downstream process protocols for many fine chemical and pharmaceutical materials occur preferentially in water, the understanding of the

WESTCHEM, Department of Pure and Applied Chemistry, University of Strathclyde, Thomas Graham Building, 295 Cathedral Street, Glasgow, G1 1XL,UK.E-mail: r.vrcelj@soton.ac.uk; Fax:+44(0) 2380 596723; Tel: +44(0) 2380596722

$\dagger$ Current address; Department of Chemistry \& Biochemistry, Chepkoilel University College, Moi University, P. O. Box 1125 - 30100, Eldoret, Kenya

\$ Current address; Department of Chemistry, University of Southampton, Highfield, Southampton, SO17 1BJ, UK physical processes that happen during water loss and uptake by the product materials is very limited.

Attempts to understand the dehydration/rehydration phenomena in molecular materials have resulted in the development of a number of kinetic models based on a range of potential mechanisms for both solid to solid phase transitions and dehydration itself. ${ }^{3}$ These historic models do play an important role in defining the rates of reaction, especially from an industrial viewpoint. However, without understanding the mechanisms of the solid state reaction or without being able to classify (which incorporates an understanding of) the process, a true definition of the nature and mechanisms of dehydration/rehydration reactions cannot be made. Based on the wider range of data available for metal/organic salt hydrates, Galwey ${ }^{4}$ has developed a "Water Evolution Type" (WET) classification. In the WET approach, the type is defined by structural, observational and kinetic criteria. A similar scheme has been proposed by Petit and Coquerel, ${ }^{4}$ based upon structural and energetic considerations. (A table summarising these two systems of classification is given as supplementary information.) As yet none of these classifications has been used to characterise purely molecular hydrates. Early work by Byrn ${ }^{5}$ has shown that some pharmaceutically based systems appear to dehydrate uniaxially, rather than isotropically. Uniaxial dehydration was associated with particular crystal structures where water has one particularly easy route (termed "channels") by which it is thought to leave the structure. Conversely, other materials were deemed "non-channel" hydrates. No other mechanistic assessment was undertaken. To fill this gap and to develop the results with the ideas of Galwey, Petit and Coquerel and Byrn we have undertaken an examination of the dehydration characteristics of several simple molecular hydrates. The present manuscript describes a study of Oxalic Acid Dihydrate as an example of a molecular hydrate with no single easy axis of dehydration. 
There are three known structures of Oxalic Acid; Oxalic Acid Dihydrate $\left(\mathrm{C}_{2} \mathrm{H}_{2} \mathrm{O}_{4} \cdot 2 \mathrm{H}_{2} \mathrm{O}\right)$, (h-OXA) and two anhydrous forms $\left(\mathrm{C}_{2} \mathrm{H}_{2} \mathrm{O}_{4}\right), \alpha$-Oxalic Acid ( $\alpha$-OXA) and $\beta$-Oxalic Acid ( $\beta$-OXA), Their crystallographic information is given in Table $1 .{ }^{6}$ Given the relative simplicity of the molecular and crystal structure, no details of the dehydration mechanism have been presented, hence the need for current study.

\section{Experimental}

\section{Preparation of samples}

Solutions of Oxalic Acid Dihydrate (Hopkin \& Williams, Essex, UK $99.8 \%$ purity), in doubly distilled water and saturated at 50 ${ }^{\circ} \mathrm{C}$ were cooled at the rate of $45^{\circ} \mathrm{Chr}^{-1}$ to $30^{\circ} \mathrm{C}$. After $3 \mathrm{~h}$ at this temperature crystalline needles of the hydrate (length $\leq 3 \mathrm{~mm}$ ) were harvested and dried. Larger single crystals for microscopic studies were grown by the slow evaporation of similar solutions saturated at $30{ }^{\circ} \mathrm{C}$. To obtain different size fractions, dried material was gently pulverized with a pestle in a mortar and then sieved through mesh sizes $(425,125,100$, and $20 \mu \mathrm{m})$. The size fractions of $425 \mu \mathrm{m}-125 \mu \mathrm{m}, 125 \mu \mathrm{m}-100 \mu \mathrm{m}, 100 \mu \mathrm{m}-20 \mu \mathrm{m}$ and $<20 \mu \mathrm{m}$ are referred to as $425 \mu \mathrm{m}, 125 \mu \mathrm{m}, 100 \mu \mathrm{m}$ and $20 \mu \mathrm{m}$. All samples were stored over a saturated solution of calcium sulphate to prevent dehydration.

\section{Differential scanning calorimetry (DSC)}

Data was collected on a DSC V2.2A Du Pont 9900 DSC. Consistent sample weights of $10 \mathrm{mg}$ were used. Samples were run at a scanning rate of $10{ }^{\circ} \mathrm{Cmin}^{-1}$ using nitrogen as a carrier.

\section{Thermogravimetric analysis (TGA)}

Non-isothermal thermogravimetry was performed using a Seiko Denshi Kogyo TG/DTA 200 instrument. The samples were heated up in open sample pans (Sample weight $5 \mathrm{mg}$ ) under dry nitrogen at a heating rate of $10{ }^{\circ} \mathrm{Cmin}^{-1}$.

\section{X-ray powder diffraction (XRPD)}

Room temperature XRPD was performed on a Siemens D500 diffractometer, utilising $\mathrm{Cu}-\mathrm{K} \alpha$ radiation, a graphite post sample monochromator and Bragg-Brentano geometry. In situ high temperature PXRD was performed at the National Crystallography Service, University of Southampton, UK, with $\mathrm{Cu}-\mathrm{K} \alpha$ radiation, flat plate geometry and an INEL C120 curved

Table 1 Crystallographic data for Oxalic Acid Dihydrate and Anhydrous forms

\begin{tabular}{lccc}
\hline & h-OXA & $\alpha$-OXA & $\beta$-OXA \\
\hline Space Group & $P 2_{1} / n$ & Pcab & $P 2_{1} / c$ \\
$a / \AA$ & 6.096 & 6.548 & 5.330 \\
$b / \AA$ & 3.498 & 7.844 & 6.015 \\
$c / \AA$ & 11.947 & 6.086 & 5.436 \\
$\beta\left({ }^{\circ}\right)$ & 108.7 & $90-$ & 115.8 \\
cell volume $\left(\AA^{3}\right)$ & 255 & 313 & 157 \\
$Z$ & 2 & 4 & 2 \\
$D_{\text {calcd }}\left(\mathrm{g} \mathrm{dm}^{-3}\right)$ & 1.56 & 1.91 & 1.91 \\
CCDC Refcode & OXACDH24 & OXALAC03 & OXALAC04 \\
\hline
\end{tabular}

detector. An Anton-Paar oven was used to generate above ambient temperatures.

\section{Atomic force microscopy (AFM)}

These were conducted with a Quesant Resolver 250 AFM, using $\mathrm{Si}_{3} \mathrm{~N}_{4}$ integral tips with a spring constant of $0.22 \mathrm{Nm}^{-1}$ in conjunction with a scan head with a maximum range of $14.6 \times$ $14.6 \mu \mathrm{m}$ for both micron scale and high resolution imaging. The scanner was calibrated with Highly Oriented Pyrolytic Graphite and muscovite mica.

\section{Thermogravimetry}

For isothermal studies, a CI Robal electronic micro-force balance was used. Data was collected electronically using an inhouse designed AD converter. Control of relative humidity $(\mathrm{RH})$ was achieved by passing dry nitrogen gas through a set of saturated solutions; $\mathrm{KOH}(8 \% \mathrm{RH}), \mathrm{CaCl}_{2}(30 \% \mathrm{RH}), \mathrm{KBr}(58 \%$ $\mathrm{RH}), \mathrm{NaCl}(75 \% \mathrm{RH}), \mathrm{KCl}(84 \% \mathrm{RH})$. Temperature control was achieved using an in-house designed bath surround.

\section{Thermomicroscopy}

A Reichert Polyvar 2 microscope was used for transmitted light microscopy, and for thermomicroscopy, a Mettler FP 5 and FP 52 system was used. Control of relative humidity for dehydration was achieved in the same method as that for Thermogravimetry. For in situ rehydration, $100 \%$ RH vapour was used.

\section{Sample control}

For comparative studies it is important to keep sample weights constant. Agbada ${ }^{7}$ has shown that an inverse relationship exists between sample weight and activation energy and is attributable to the greater self-cooling effect of larger sample mass on endothermic reactions.

\section{Results and discussion}

\section{Crystal growth and morphology}

The morphology of crystals grown by slow evaporation was defined by using goniometric measurements in association with a stereographic projection of the crystal. The principal face on these plate-like crystals was identified as the (001) face and the smaller adjacent faces, the corresponding $\{100\}$ and $\{110\}$ faces (Fig. 1).

\section{Dehydration product}

PXRD confirmed that the principal product of Oxalic Acid dehydration is the monoclinic anhydrous form ( $\beta$-OXA).

\section{Structural assessment}

The h-OXA structure is a relatively close packed layered structure (for a hydrated form), with planes of Oxalic Acid molecules lying parallel to the (101)) plane (Fig. 2a). Between and lying parallel to these planes are layers of water molecules. The structure supports a network of cross-linked hydrogen-bonds, with the water molecules connecting to the hydroxyl and 


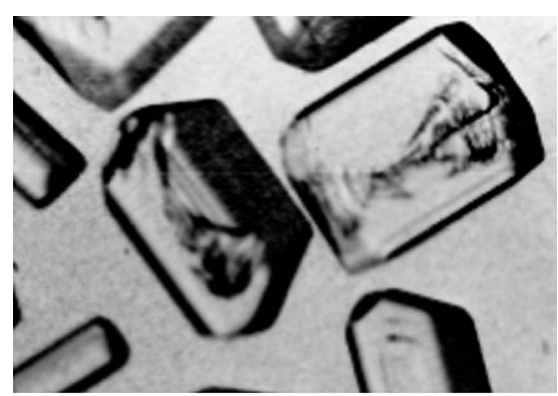

(a)

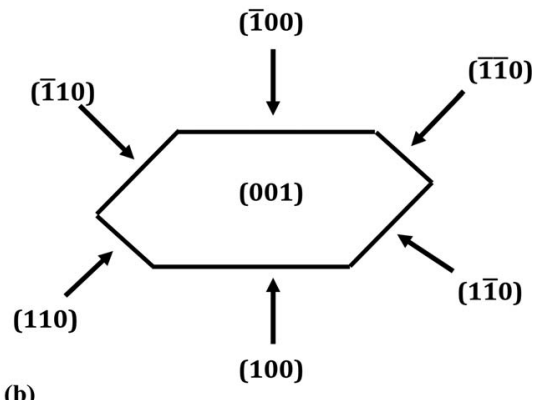

(b)

Fig. 1 (a) 'As crystallized' h-OXA crystals from solvent evaporation and (b) their morphology.

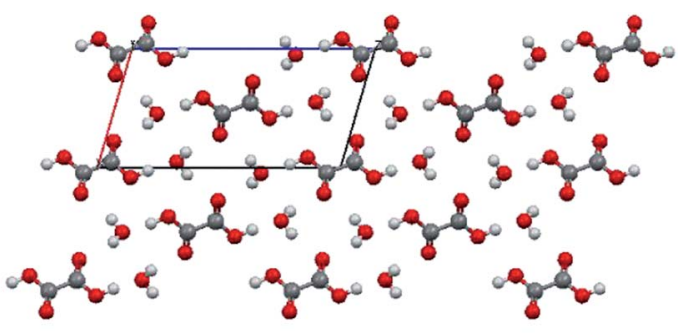

[100]

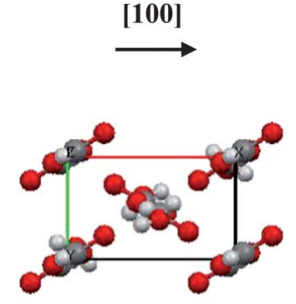

h-OXA

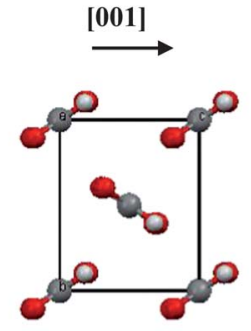

B-OXA
Fig. 2 (a) The extended packing of h-OXA as viewed along [010] (b) Similarity of molecular packing between h-OXA along [100] and $\beta$-OXA along [001].

carboxyl groups of the Oxalic Acid entities. Each Oxalic Acid molecule is co-ordinated by six water molecules and conversely, each water molecule co-ordinated by three OXA molecules. There is no difference between the two water molecules from a co-ordination and hydrogen bonding viewpoint. The hydrogen-bonding schemes create interpenetrant planar networks of hydrogen-bonds that lie parallel to the (110) and
(110) There appears to exist an easy direction of dehydration, [010], however, this channel diameter is at largest $\sim 1 \AA$, which is far too small to permit water molecules to move and it is extremely unlikely that this is a real channel for movement of water molecules.

$\beta$-OXA is formed of chains of hydrogen-bonded Oxalic Acid molecules running along [100], forming a close packed arrangement.

There exists a structural similarity between the two forms (identified using $\mathrm{XPac}^{8}$ ), with the h-OXA [100] lattice vector closely resembling the $\beta$-OXA $[\overline{1} 0 \overline{1}]$ lattice vector. In addition to the direct structural relationship, the molecular packing arrangement as expressed on the h-OXA (001) face mimics that of the $\beta$-OXA (100) face (Fig. 2b).

\section{Dehydration}

\section{Optical microscopy}

In reflection mode, the crystal surface initially appears relatively smooth (Fig. 3a), the major features that are clearly visible are gross macrosteps, either from the crystal growth process itself or from removal of the crystal from the mother liquor. As the dehydration initiates (Fig. 3b, after $30 \mathrm{~min}$ ), contrast at edges of macrosteps is enhanced and surface changes initiated at these imperfections are clearly visible. This behaviour is common across all solid phase reactions, where any edges, corners or imperfections in crystal surfaces and structures enhance reactivity and decomposition of solids. This is mimicked in larger scale observations of the dehydration of the present crystal,
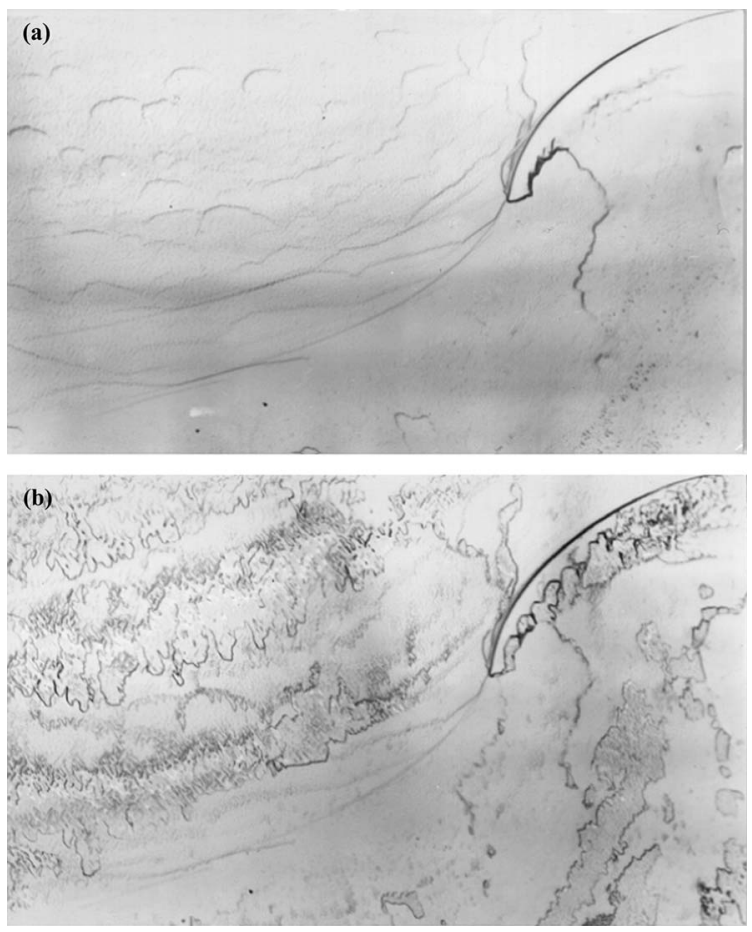

Fig. 3 Optical reflection micrographs showing surface changes of hOXA during dehydration at $35{ }^{\circ} \mathrm{C}$ (magnification x200); (a) smooth surface before dehydration, (b) removal of water from surface after $30 \mathrm{~min}$. 
where the dehydration occurs predominantly at the corners and apexes of the crystals and only reaches the centre of the (001) face at the end of the dehydration process.

Higher resolution transmission microscopy images of the dehydrating (001) surface show that large scale decomposition nucleates at some point on the surface and is composed of an array of micro-crystallites of the product phase, radiating from that point on the surface (Fig. 4), with a reaction front surrounding it.

\section{Atomic force microscopy}

A low resolution AFM image (Fig. 5a) of the hydrated h-OXA (001) faces shows a relatively rough surface. The most interesting parts of the image are the lines of structures lying parallel to the crystallographic $b$ axis. These cannot be dislocation etch pits as they are higher than the general surface surrounding them.

The surface of dehydrated h-OXA (Fig. 5b), shows three features worthy of note; firstly the overall variation in surface topography increases from $400 \mathrm{~nm}$ to $850 \mathrm{~nm}$ as might be expected from a large scale roughening of the surface accompanying the dehydration. Secondly, step changes in height along [001] on the crystal surface indicate a relative movement has occurred between parts of the (001) face. Lastly, although smaller localised imperfections have been removed, there are still parts of the image that show some co-linearity with the b-direction. In general, however, the surface is very smooth and clean.

\section{Thermal studies}

Isochronal TGA shows a steady loss of weight of $\sim 29 \%$ between $60{ }^{\circ} \mathrm{C}$ and $110^{\circ} \mathrm{C}$ and then further mass loss to zero between 110 ${ }^{\circ} \mathrm{C}$ and $190{ }^{\circ} \mathrm{C}$, indicative of a simple two step decomposition process of dehydration followed by decomposition into gaseous products. The associated DSC trace yields an enthalpy of dehydration value of $568 \mathrm{Jg}^{-1}$ corresponding to a water value of $\mathrm{n}$ $=1.7,{ }^{9}$ rather than the expected 2.0.

\section{Optical thermal studies}

Hot stage microscopy shows the process in detail. Fig. 6a shows the (001) surface of a freshly harvested transparent h-OXA crystal. With increasing temperature, the crystal dehydrates and becomes opaque (Fig. 6b). At higher temperatures,

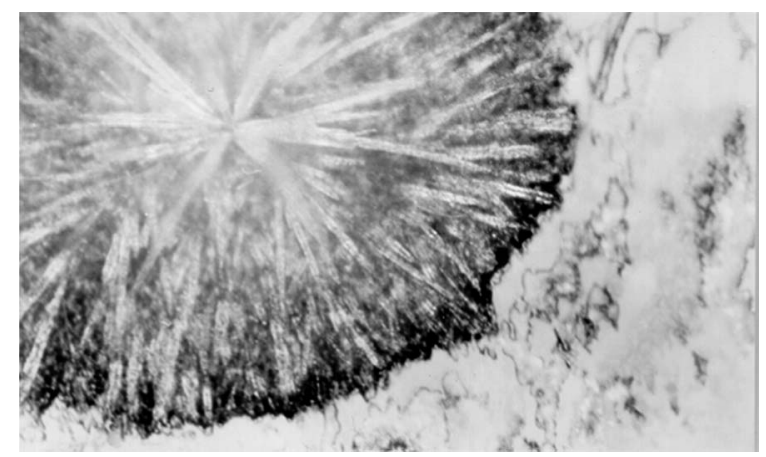

Fig. 4 Optical transmission micrographs of the dehydration of h-OXA at higher magnification (x400), showing advancing phase boundary. crystallisation began to occur on the upper cover slip of the heating stage, indicating that sublimation and recrystallisation of Oxalic Acid was occurring (Fig. 6c). This process continues as the original crystal gradually reduced in size and disappeared by 165 ${ }^{\circ} \mathrm{C}$. Finally, at approximately $210^{\circ} \mathrm{C}$, all the sublimed crystallites disappear.

These experiments combine to show that the decomposition has three distinct elements: Initial dehydration and sublimation (65 to $120{ }^{\circ} \mathrm{C}$ ), competing mechanisms of sublimation and decomposition (120 to $190^{\circ} \mathrm{C}$ ) and finally decomposition of the sublimed crystals (above $190^{\circ} \mathrm{C}$ ).

The clear evidence for sublimation can explain both the underestimation of the amount of water lost from the DSC data and the AFM images of a smooth surface. In the case of the DSC data, if, in addition to the water loss, OXA molecules are being lost from the solid, then this immediately explains why the underestimation takes place. For the AFM image, as sublimation occurs from the surface, this acts in an ablative manner, removing surface damage and cleaning the remaining layer.

\section{Dehydration at the molecular level}

The process of dehydration in Oxalic Acid is controlled by a number of competing factors. The intrinsic ability of h-OXA to lose water (breaking of hydrogen bonds) and the structural relationships between the hydrated structure and the anhydrous product. In addition to this, there are the extrinsic factors, such as the role played by the gross physical characteristics of the crystal - the morphology and any defects within the structure.

Two fundamental questions arise; 1) How does the water escape from the h-OXA structure so easily and 2) Why does rehydration occur with relative ease?

The answer to question 1 lies in the crystal structures of hOXA and $\beta$-OXA. The faces of h-OXA are $\{100\},\{001\}$ and $\{110\}$ types, and the water molecules lie on the (10) $\overline{1})$ plane. This plane intersects all the available faces, so all the water molecules have an accessible surface from which they can depart the parent crystal. However, once the h-bonds are broken and the outermost water molecules leave the structure, there is apparently no method (even if the h-OXA structure retains its coherence and stays as an un-hydrated h-OXA structure) by which the next set of water molecules can leave. Such an interpretation is consistent with the lack of observations of dehydration patterns at the crystal surface especially along the b-direction. Although some further water molecules will be released as the OXA molecules sublime, this will not account for the totality of the process - fully dehydrated material can be made and handled.

Examining the h-OXA structure can give answers to this conundrum. If the water molecules are removed from the structure (Fig. 7) the only remaining molecular interactions are short order van der Waals forces that run in long chains within the (001) plane, parallel to [010]. Along [001], there are no interactions, simply voids. This implies that when water leaves the structure, the dehydrated structure will collapse along [001], as observed by AFM, effectively forming a discontinuity that is parallel to the (001) face, a large scale defect structure. This disassociation will cause disruption across the $\{100\}$ and $\{110\}$ faces, making available space along which water can diffuse out once detached from the crystal structure. In the case of the (001) 


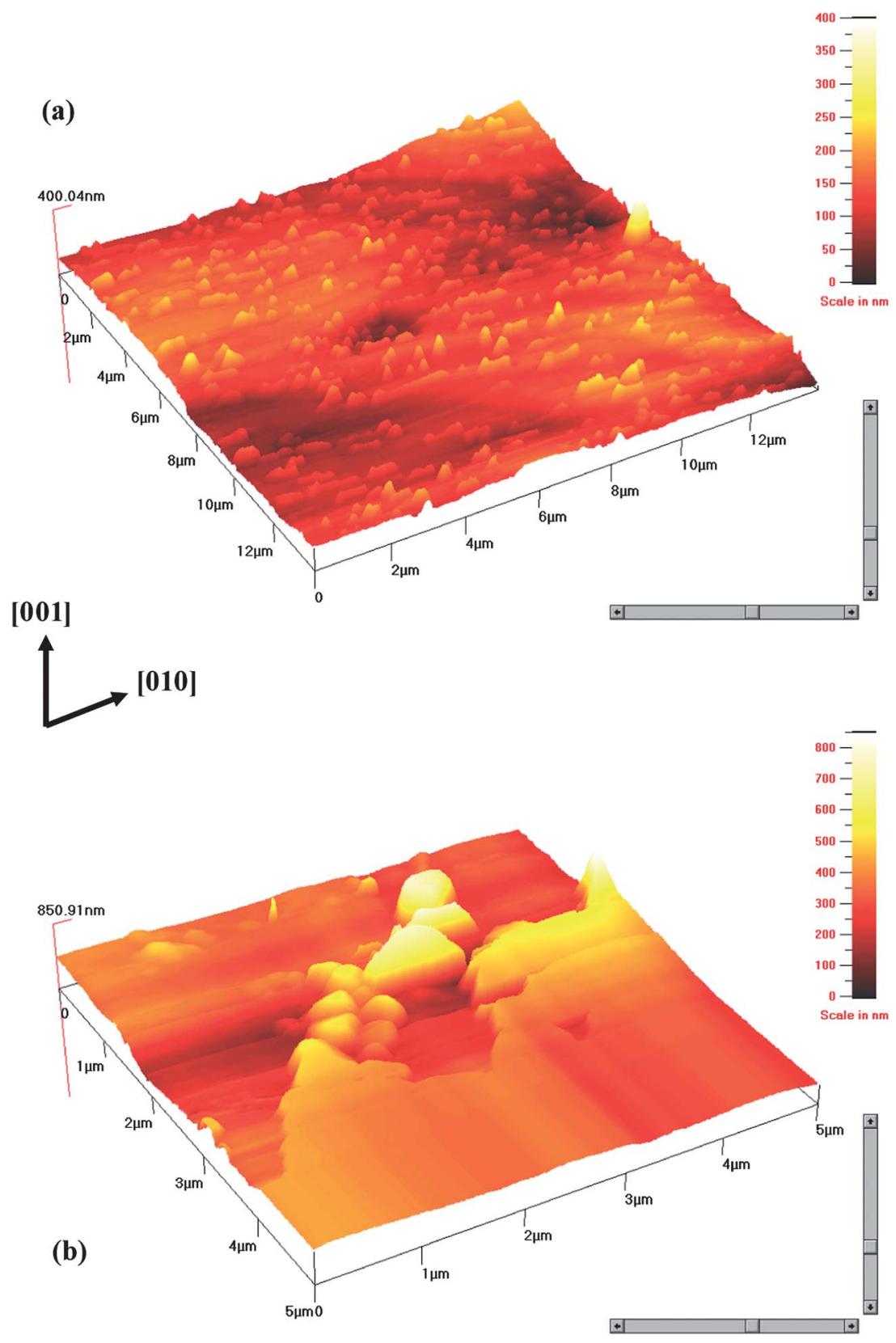

Fig. 5 AFM images of the (001) surface of h-OXA; (a) hydrated (b) dehydrated.

face, the remaining van der Waals interactions keep some semblance of the original structure available within the (001) plane, but the planes of molecules themselves undergo disruption. Effectively the large scale (001) planes are severely degraded - effectively unzipping the structure along the (001) planes.

The similarity of the h-OXA [100] and $\beta$-OXA [1]01] directions can then act as a nucleation site for growth of $\beta$-OXA. The van der Waals forces (parallel to h-OXA [010]) that remain in the residual (waterless) h-OXA structure means that local order will be retained.

This idea of preferential disassociation is supported by in situ PXRD (Fig. 8). Where, during the dehydration process, the peaks associated with the (002) and (004) diffraction planes, disappear much more quickly (within $35 \mathrm{~min}$ at $40{ }^{\circ} \mathrm{C}$ ) than the remainder of the available diffraction peaks (complete at $60 \mathrm{~min}$ at $40{ }^{\circ} \mathrm{C}$ ). Within the set-up of the experiment, this change in diffraction intensity during the dehydration experiment can only be attributed to a change in the relative scattering power of the (001) planes, i.e. the (001) planes lose coherence at an earlier stage than the remaining lattice.

This combination of factors also accounts for the optical microscopy and AFM observations. In the case of optical microscopy, the nucleation of any given site on the (001) face will be then surrounded by further growth of the resultant phase, but with no obvious water loss at that surface. For AFM, the smoothness of the surfaces can be attributed to the sublimation of the surface layers of both water and OXA, but the relatively large changes in surface levels of the (001) will be caused by the 

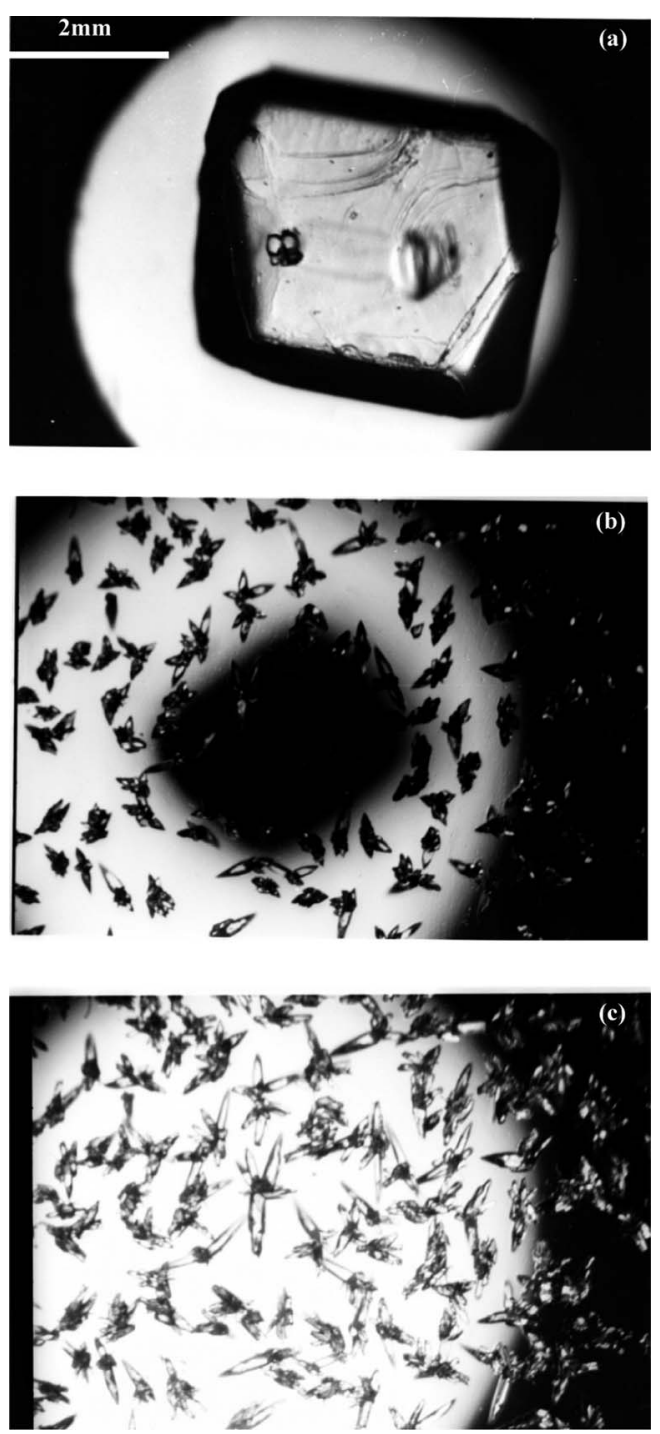

Fig. 6 Optical Micrographs of the decomposition of h-OXA, viewed along [001]; (a) $28^{\circ} \mathrm{C}$ (b) $135^{\circ} \mathrm{C}$ (c) $165^{\circ} \mathrm{C}$.

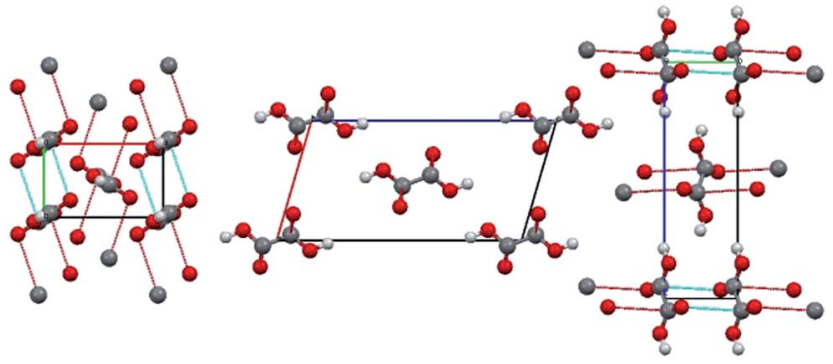

(a)

(b)

(c)

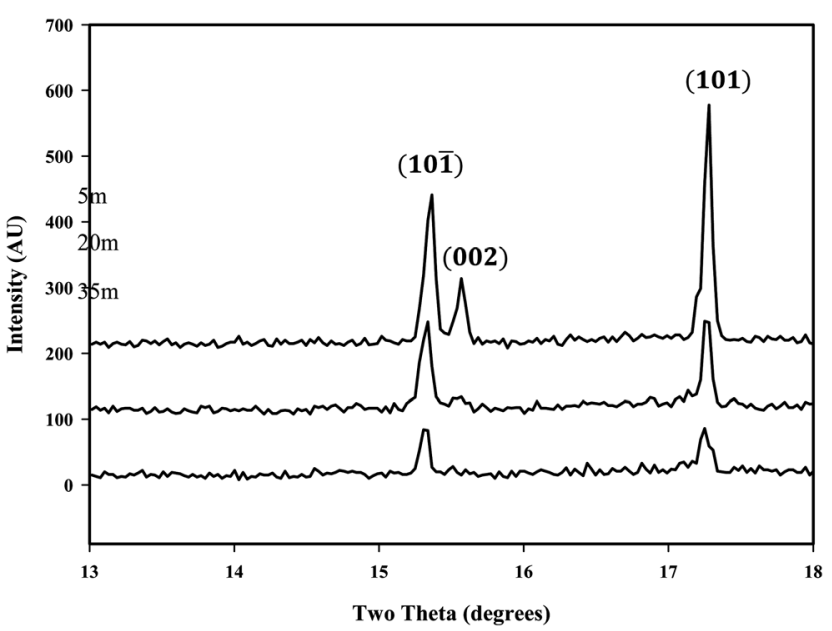

Fig. 8 In situ PXRD showing the preferential disappearance of the (002) planes at $40{ }^{\circ} \mathrm{C}$.

The in situ PXRD experiments also show no extraneous diffraction peaks, indicating that there is no intermediate crystalline phase (at levels of $\sim>1 \%$ ). Also there no change in the general background, implying that there does not exist any gross amorphous intermediate phase between h-OXA and $\beta$-OXA, however, this data does not have good enough counting statistics required to accurately quantify this for relatively low levels of intermediate amorphous phase.

Although there is a topotactic relationship between h-OXA and $\beta-O X A$, this will not extend to the medium and long range the overall difference between the structures means that as the disassociation occurs, small areas of $\beta$-OXA will form (and crystallisation further enhanced by effective annealing of the product) but a disruptive boundary layer will occur, leaving physical gaps, through which the water can leave the entire structure - this is shown well in Fig. 3, where the unreacted and reacted areas show little contrast, but the boundary layer between them is much more disrupted and thus of greater contrast.

\section{Rehydration}

A general observation of the rehydration of $\beta$-OXA is that at ambient conditions, it occurs rapidly, often within the time taken to transfer a sample from one piece of equipment to another. For example, transfer of material recovered from either in situ PXRD or isothermal gravimetric measurements always yields a material that has partially rehydrated. This means that the most consistently replicable rehydration experiments are those which can be performed in situ, such as optical microscopy and isothermal gravimetry.

\section{Rehydration optical microscopy}

Microscopic examination of the early stages of dehydration and rehydration shows interesting features which appear to be wholly reversible. The original crystal (Fig. 9a) darkens to near opacity after $1 \mathrm{~h}$ dehydration (Fig. 9b), but after rehydration for one hour, (Fig. 9c), the crystal looks identical to the starting image. collapse of the (001) face during dehydration. These structural changes show how a topotactic relationship between the two forms affects the dehydration product. 

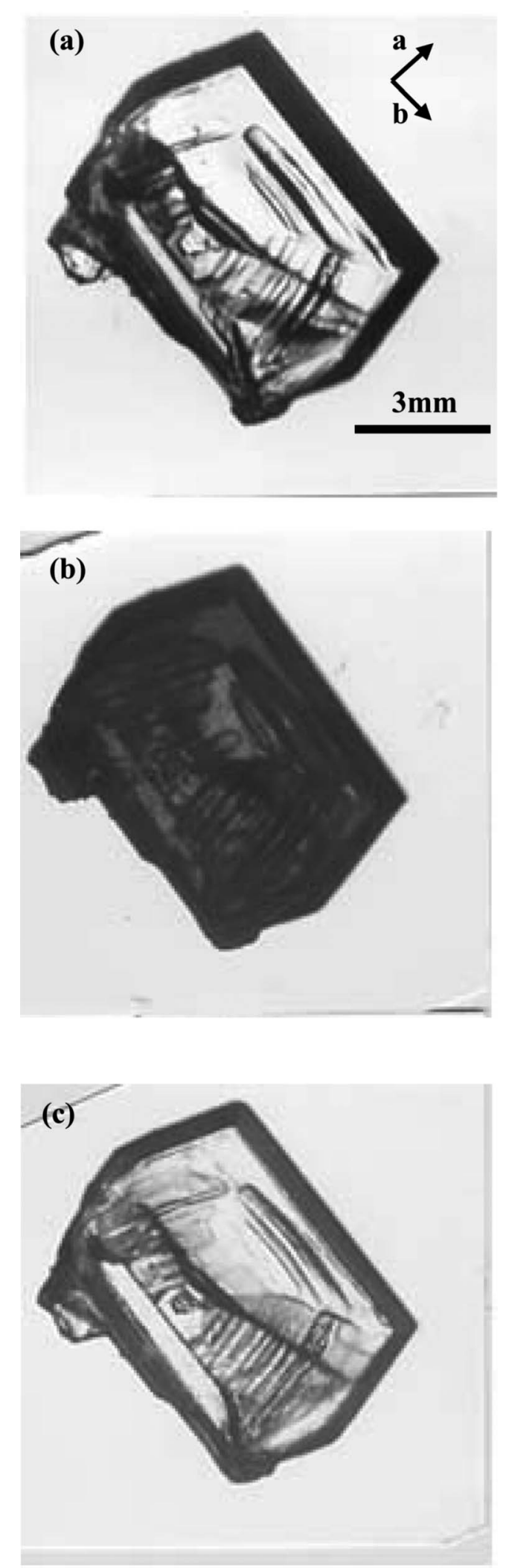

Fig. 9 Optical transmission micrographs of the partial dehydration and rehydration of h-OXA; (a) $0 \mathrm{~min}$, (b) $75 \mathrm{~min}$, (c) $165 \mathrm{~min}\left(T=26^{\circ} \mathrm{C}\right)$.
A higher magnification study shows a different story. The original surface (Fig. 10a) is relatively smooth, with very few macrosteps and some surface debris. The rehydrated surface is covered with small nucleation centres some of which have grown into h-OXA crystals (Fig. 10b).

The morphology of these crystallites is the same as the parent face, with the (001) facet evident. Although the crystals have the same morphology as the parent, it is of interest to note that they lie either parallel to the parent face or at an angle of $\sim 19^{\circ}$. Clearly, the early stages of rehydration can be viewed as part of a nucleation and growth process of h-OXA.

The growth can only be through localised vapour phase or solution growth onto the crystal surface. i.e. the available water vapour dissolves the surface $\beta$-OXA and re-deposits it as crystal growth of h-OXA. This will account simply for those crystallites that are arranged parallel to the original surface structure, the $\beta$ OXA is effectively dissolved back and growth continues on the bulk of the original h-OXA crystal. For those that lie at an angle to the original surface, we can attribute this to residual areas of $\beta$ OXA that do not fully redissolve but act as nucleation sites. If a comparison is made of the $\beta$-OXA (100) face and the h-OXA (001) face (Fig. 2b), it can be seen that the molecules are out of alignment by $\sim 9^{\circ}$, if some residual $\beta$-OXA exists, then any $h-$ OXA that grows onto it will also readjust by $\sim 9^{\circ}$, to achieve an energetically favourable structure. Of course, this $\sim 9^{\circ}$ can fall either one side or another of the $\beta$-OXA structure, either yielding
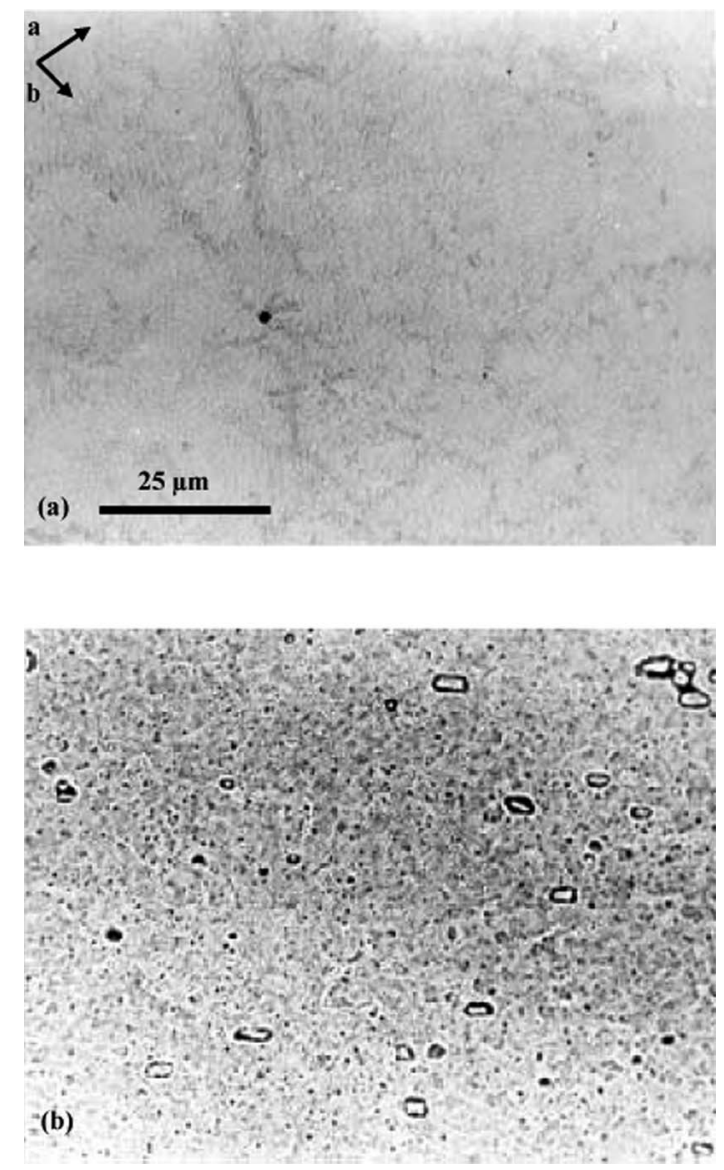

Fig. 10 Optical reflection micrographs of the partial dehydration and rehydration of h-OXA (001\} surface; (a) $0 \mathrm{~min}$, (b) $150 \min \left(T=26^{\circ} \mathrm{C}\right.$ ). 
crystal that are again oriented with the original direction, or those that are seen to be at an angle of $\sim 18^{\circ}$ to the original face and as shown in Fig. 10b.

This also implies that in addition to topotaxy, there may be elements of templating and minor epitaxy that occur during the rehydration process, through a residual $\beta$-OXA layer on the surface of the parent h-OXA crystal.

This answers question 2 posed earlier. The ease with which $\beta$ OXA rehydrates is a measure of the ease of recrystallization of hOXA through a vapour reaction. The close structural relationships, allied to the ease with which OXA sublimes, means there will be always be some rehydration occurring.

\section{Kinetic studies}

\section{Effect of temperature}

Typical isothermal fractional dehydration $(\alpha-t)$ curves for $h-$ OXA are sigmoidal in shape and have a deceleratory form - the $0 \%$ RH examples are shown in Fig. 11. Dehydration of h-OXA occurs rapidly, with the slowest process $(2 \mathrm{~h})$ occurring in the lowest temperature experiments $\left(40^{\circ} \mathrm{C}\right)$.

Kinetics analysis ${ }^{10}$ shows that the best fitting model (for all dehydration studies in this paper) is the Two Dimensional Phase Boundary reaction process, $\mathrm{R}_{2}(\alpha)$.

$$
1-(1-\alpha)^{1} / 2=k t
$$

The regression coefficients ranged between $0.992-0.999$ for the different runs, however, the second best model (the 1-Dimensional diffusion reaction model) has similarly good regression coefficients $0.988-0.990$. As has been noted by Galwey, ${ }^{4}$ any one of the kinetic models will account for the rate of advance of the interface, but without any real determination of the mechanism, such as nucleation and growth or the role of concurrent diffusion processes. The choice of which kinetic model is to some degree unimportant as for any well-fitting model, the calculated kinetic parameters will be similar whichever model is used. The mechanism by which the water disassociates from the hydrate lattice and is lost through the resultant structure will dominate the

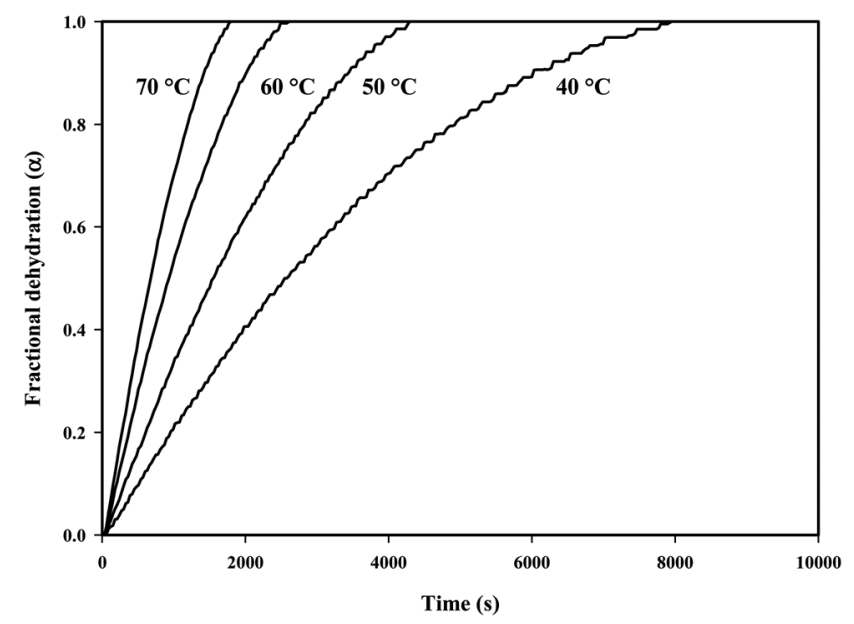

Fig. 11 Typical set of isothermal gravimetric measurements of the dehydration of h-OXA. The samples have particle size range $425 \mu \mathrm{m}$ and the runs were performed at $0 \% \mathrm{RH}$. process. In the present case the restructuring of the waterless $h$ OXA lattice into the $\beta$-OXA form, via collapse of the (001) planes and the loss of the water molecules (via a diffusion process) through the resultant deformed structure comprise a dual process. Nevertheless, the ideals behind model fitting may be valid and give some measure of the process as a whole, rather than a true understanding of the dehydration itself.

The $\mathrm{R}_{2}(\alpha)$ model Arrhenius plot yields an activation energy $\left(E_{a}\right)$ of $53(3) \mathrm{kJmol}^{-1}$ for $425 \mu \mathrm{m}$ particle size. The average value for all experiments was 51(3) $\mathrm{kJmol}^{-1}$ which lies within the values of $50-60 \mathrm{kJmol}^{-1}$ obtained from TGA by Sudhakaran and Radhkrishnan Nair, ${ }^{11}$ but lie below that of Guarini and Dei, ${ }^{11}$ who find higher values for both Activation Energy (82 \pm 2 $\left.\mathrm{kJmol}^{-1}\right)$ and enthalpy of dehydration $\left(103 \pm 3 \mathrm{kJmol}^{-1}\right)$, but it is comparable to other organic hydrated systems, for example cimetidine hydrate. ${ }^{12}$

An observation of the kinetic data is that there appears to be a stepwise dehydration. Fast dehydration experiments yield smooth monotonically increasing curves, but as the temperature of the experiments decreases, the appearance of small steps in the dehydration process becomes more evident - in Fig. 11, this is clear for the run performed at $40{ }^{\circ} \mathrm{C}$, when the mass fraction dehydrated is greater than 0.6 - these steps in mass are much greater than the resolution of the used balance and are not artefacts of the experimental system. This "holding" of the water is easily explained by the lack of "channels" in the structure of hOXA and the fact that to lose water, it must develop relatively large scale defects in the parent body. As the water disassociates from the h-OXA lattice, it will be trapped between that and the restructuring $\beta$-OXA. Once sufficient pressure builds, and the $\beta$ OXA finally either physically leaves the parent body (simply falling off), or undergoes sufficient annealing to minimise its surface area, the water can then be released. With higher temperatures, this process occurs much more quickly. Such large scale features are seen, for example with the surface disruption observed with the AFM (Fig. 5b). The question also arises of how to interpret these from a modelling perspective. Should the curve be fitted as an "average", or should the individual steps be analysed separately (should each individual step be considered as an independent dehydration event). Attempts to model the small steps have been made, but unrealistic results are obtained, which is not surprising, considering that the process of step removal is not a measurement of an interface advance, but of a different process. As such, we continue to use the "average" model to examine the kinetic data. However, it is possible to simulate the effect by covering an h-OXA crystal with an impermeable layer (glycerol). The associated dehydration curve (Fig. 12) shows the same features as the smaller stepped pure h-OXA, but with longer "quiet" periods and larger step sizes. Simply a reflection of the difficulty of the water permeating through an impenetrable layer.

\section{Effects of particle size and relative humidity and gas flow rates}

The effects of particle size on the kinetic parameters can be seen in Fig. 13. As with the variable temperature runs (Fig. 11), steps are seen, and as expected, they are more pronounced for larger particle size. Similar step-wise curves are found for increased $\%$ $\mathrm{RH}$ runs. By examining the $\mathrm{E}_{\mathrm{a}}$ data generated (Fig. 14), it is 


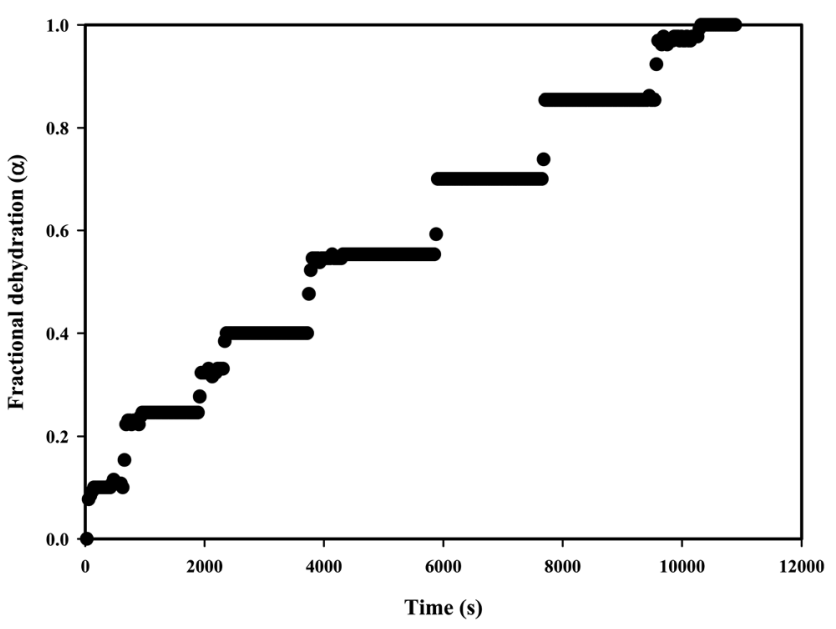

Fig. 12 The dehydration of a single crystal of h-OXA, with the surface covered with a thick layer of glycerol.

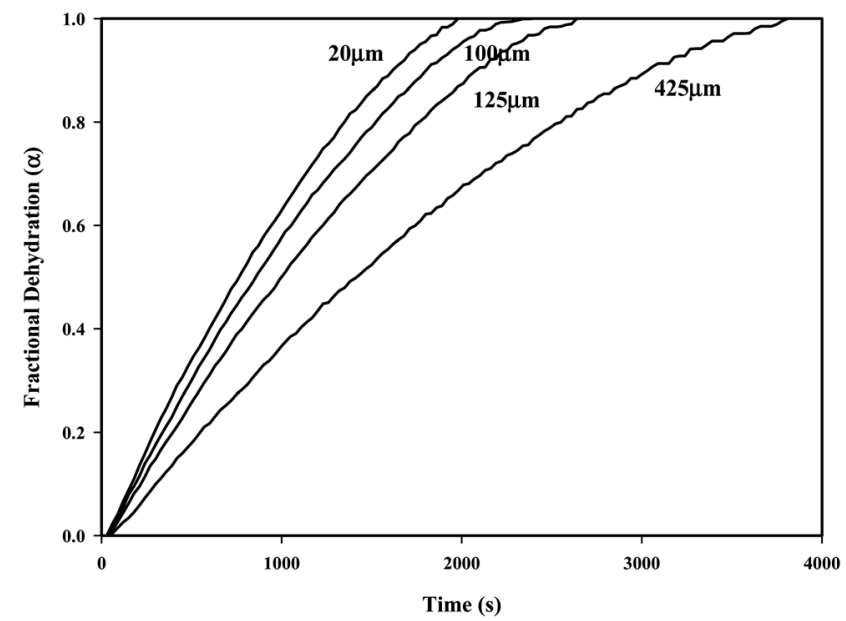

Fig. 13 Effect of particle size on dehydration of h-OXA. The runs are at $50{ }^{\circ} \mathrm{C}$ and $0 \% \mathrm{RH}$.

possible to see that at low $\mathrm{RH}$, the difference in particle size does not affect the $E_{a}$ of the process, indicating that the same mechanism of lattice water loss and lattice disruption occurs in a similar way. However, as the $\% \mathrm{RH}$ increases, the dispersion of the values of $E_{a}$ becomes larger. This shows how differences in particle size and will affect the observed dehydration profiles given that the same mechanism of water loss is occurring. The overall trend to greater $\mathrm{E}_{\mathrm{a}}$ is surprising, as larger particle size and higher $\% \mathrm{RH}$ should, intuitively, make dehydration less easy to initiate. However, the Smith-Topley ${ }^{13}$ effect was not observed.

It is also possible to observe changes due to the purge gas flow rate (Fig. 15). In a similar and expected manner, increasing the purge gas flow rate decreases the time taken for dehydration and decreasing the rate enables the step-wise nature of the process to be seen.

\section{Rehydration reaction}

The solid-state dehydration reaction of h-OXA is a reversible reaction. The dehydration yield product, $\beta$-OXA is hygroscopic.

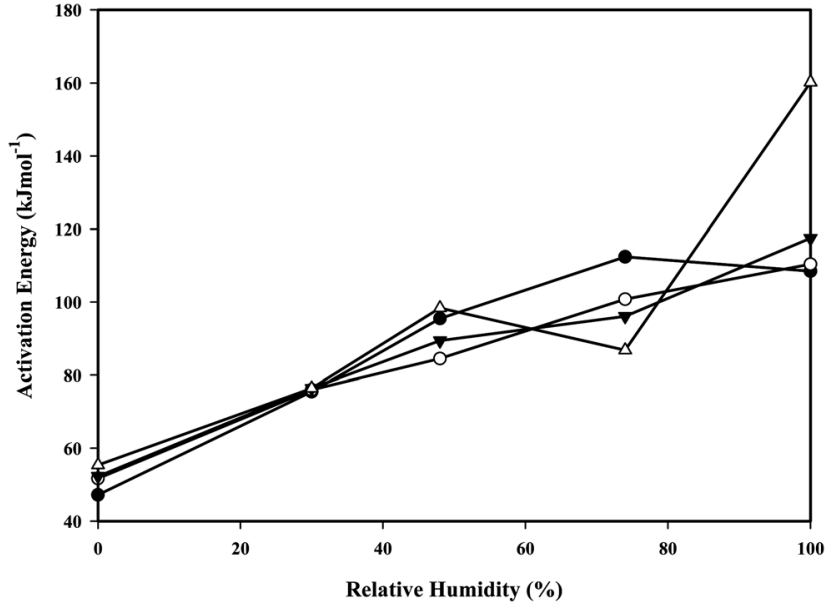

Fig. 14 The effect on activation energy with particle size and relative humidity. The plots indicate the particle size ranges as follows $425 \mu \mathrm{m}$ $(\triangle), 125 \mu \mathrm{m}(\boldsymbol{\nabla}), 100 \mu \mathrm{m}(\bigcirc), 20 \mu \mathrm{m}(\bullet)$.

Its exposure to ambient humidities results in a rapid rehydration to yield h-OXA, with rehydration complete within $3 \mathrm{~h}$. Although the dehydration/hydration process is reversible, the processes that occur are not similar. As mentioned previously, the formation of the hydrated phase requires a nucleation and growth of the hydrated phase, either from the vapour or liquid phase.

\section{Thermogravimetry}

Isothermal gravimetric studies show that the kinetics of rehydration follow a deceleratory pattern; with successive dehydrations and rehydrations exhibiting a hysteresis (Fig. 16). Fitting the data to the standard kinetic models showed the best fit to be a Three Dimensional Phase Boundary $\mathrm{R}_{3}(\alpha)$ model.

$$
1-(1-\alpha)^{1 / 3}=K t
$$

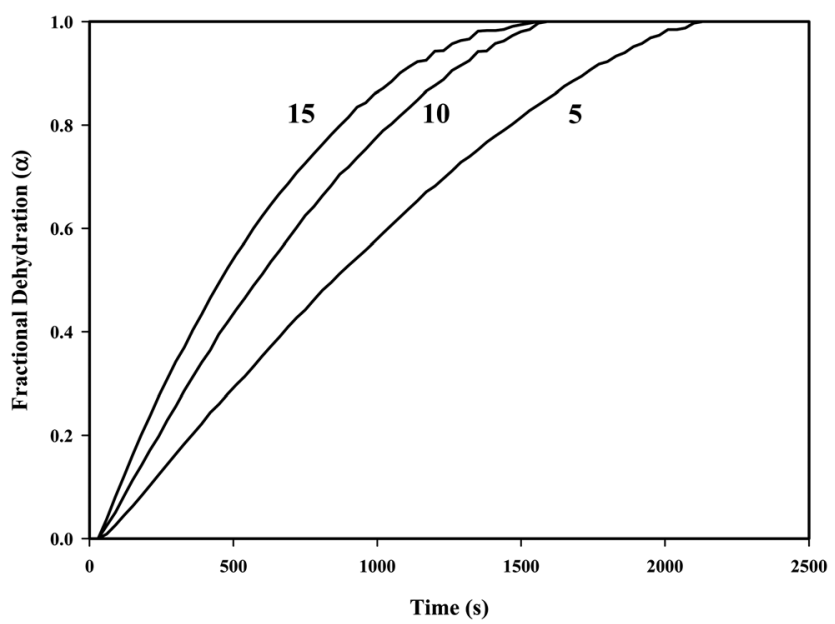

Fig. 15 Effect of purge gas rate on dehydration. The samples have particle size range $425 \mu \mathrm{m}$, the runs were performed at $30^{\circ} \mathrm{C}, 0 \% \mathrm{RH}$ with gas purge rates of 5,10 and $15 \mathrm{~cm}^{3} \mathrm{~min}^{-1}$. 


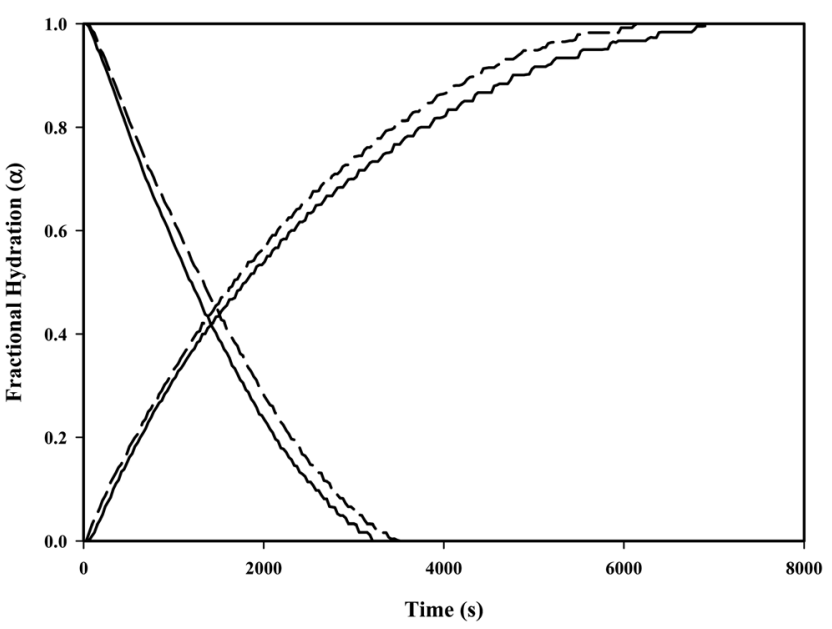

Fig. 16 Hysteresis of the dehydration/rehydration process. The initial dehydration (dehydration temperature $62{ }^{\circ} \mathrm{C}$ ) and rehydration (rehydration temperature $26^{\circ} \mathrm{C}$ ) cycle is a full line and the second cycle is shown as a broken line (particle size range $425 \mu \mathrm{m}$ ).

Given that earlier observations show that recrystallization occurs, it is not surprising that this particular model fits the data well. In particular, given the process that is occurring, it may well be a viable statement to say that this form of modelling does aid understanding of the rehydration process, as opposed to dehydration.

Thermogravimetry also confirms that it takes much longer for the sample to rehydrate than to dehydrate. The rehydration also shows what appears to be "stepped" periods, similar to those for the dehydration process, but are more pronounced. This we can also ascribe to the growth process, during the recrystallization of the outer surface. A fully rehydrated form is created and must disassociate from the residual anhydrous material, before that can undergo a recrystallization. This would account for the longer step period and larger step size, compared to the dehydration step process.

The hysteresis in the process is of note, in that the rate of rehydration increases, which can be intuitively explained by physical breakdown during dehydration, yielding a greater surface area to undergo the process. In contrast, the rate of dehydration decreases, whilst it might have been expected to increase. However, as the rehydration is a recrystallization process, it will also form less well defined crystals, with large areas of inclusions and defects, as well as areas of incomplete rehydration (as shown earlier). These factors, in particular the retention of the $\beta$-OXA phase, will mitigate the effects of general particle size reduction.

This raises a further question that cannot be answered at the present time. Why is the dehydrated product $\beta$-OXA, rather than $\alpha$-OXA? Nominally $\beta$-OXA is thermodynamically the more unstable of the two anhydrous forms. Although there is little hard data, the fact that large single crystals of $\alpha$-OXA can be grown from acetic acid and ether solutions ${ }^{\mathbf{1 4 , 6}}$ (and is the basis for other studies ${ }^{15}$ ), whereas $\beta$-OXA seems only to have been grown from sublimation at higher temperatures ${ }^{6}$ indicates that the $\alpha$ OXA is the more stable anhydrous form at ambient temperatures. Is the dehydration process generating a variation of
Ostwald's rule of stages for polymorphism? Or is this because there is a better structural relationship between h-OXA and $\beta$ OXA?

\section{Conclusion}

A more comprehensive approach to the analysis of the dehydration and rehydration processes in Oxalic Acid has shown that the more simplistic methods previously used do not give an unambiguous picture of what is happening even in simple systems. It is possible now to define the competing processes more completely; general physico-chemical effects, effects of crystal structure, effects of particle size, effects of $\mathrm{RH}$ and the rehydration process itself.

The planar nature of the dehydration means that the crystals dehydrate isotropically and at higher temperatures, This ease of dehydration is exacerbated at higher temperatures by sublimation of the crystal surface. The planar nature also lends itself to some disruption in the lattice (where the water is lost), but also to partial coherence of the structure and topotaxy. In particular this can be seen in rehydrating crystal samples.

With a better view of the physico-chemical processes that are occurring within the material, is it now possible to better appreciate data that is collected from a wider variety of methods, in particular kinetic data. While is it still difficult to truly ascribe real thermodynamic variables to kinetic data, it is possible to see how this can also yield good physical information to support the understanding of how materials behave - in particular the stepwise process during dehydration and rehydration.

In terms of correlation with the existing classifications of solid state reaction type h-OXA behaves in an interesting manner. It is not a channel hydrate in the terminology of Byrn, but the crystallographic packing does affect the dehydration process. In the Galwey scheme, the process has elements of both WET 2 and WET 3, fitting closely to both descriptions, but into neither fully. In this case should a new WET number be defined or ought it lie between the two existing classifications, leading to a WET 2.5 classification for the dehydration of h-OXA?

Similarly, in the scheme of Petit and Coquerel, the classification initially appears much clearer, with the process occurring through a type I method, with a two stage co-operative loss of water, but with some structural inter-relationship. As with the WET scheme, this process does not exactly fit into any of the current classifications.

The inability of the current classification schemes to define the dehydration process of Oxalic Acid dihydrate indicate the lack of full data in the area of dehydration. With better data and clearer observations, it is likely that a more complete scheme (most likely based on either the Galwey or Petit and Coquerel method, or a combination of both) will evolve. Byrn's channels do not exist within h-OXA in a classic sense, but the crystallographic packing of the water within a system does affect the way in which dehydration occurs and By implication, this classification could well be extended to planes and 3dimensional networks of dehydration (and desolvation routes). If these can be identified, they would almost immediately become part of the understanding of the general classification and could possibly give a better physical basis for the characterisation of dehydration processes. 


\section{Acknowledgements}

We acknowledge the kind support of GlaxoSmith Kline for their financial support of this work. In particular MOO wishes to express his gratitude for the firm's financial support of his MSc. and subsequent $\mathrm{PhD}$ studies. $\mathrm{He}$ also thanks his employers, Moi University, Kenya for according him study leave to allow him to undertake these Studies. We also wish to thank Mr David Merrifield of Glaxo Smith Kline and Professor A. K. Galwey, Queens University, Belfast for their kind interest in this work and for many stimulating and helpful discussions on this topic.

\section{References}

1 F. H. Allen, Acta Crystallogr., Sect. B: Struct. Sci., 2002, B58, 380388.

2 L. Infantes, L. Fabian and W. D. S. Motherwell, CrystEngComm, 2007, 9, 65-71; I. Nobeli and S. L. Price, J. Phys. Chem. A, 1999, 103, 6448-8457; P. Macchi, N. Casati, W. G. Marshall and A. Sironi, CrystEngComm, 2010, 12, 2596-2603.

3 M. E. Brown, D. Dollimore and A. K. Galwey in Reactions in the Solid state; Comprehensive Chemical Kinetics, Elsevier, Amsterdam vol. 22, 1980; A. K. Galwey and M. E. Brown, Thermal
Decomposition of Ionic Solids, Elsevier, Amsterdam, 1999; W. E. Garner in Chemistry of the Solid-state, Ed. W. E. Garner, Butterworth, London, 1955, 213; P. W. Jacobs and F. C. Tompkins in Chemistry of the Solid-state, Ed. W. E. Garner, Butterworth, London, 1955, Chapter 7.

4 A. K. Galwey, Thermochim. Acta, 2000, 355, 181-238; S. Petit and G. Coquerel, Chem. Mater., 1996, 8, 2247-2258.

5 P. R. Perrier and R. S. Byrn, J. Org. Chem., 1982, 47, 4671-4676.

6 Oxalic Acid Dihydrate e.g. CCDC Refcode OXACDH24; Y. Wang, C. J. Tsai, W. L. Liu and L. D. Calvert, Acta Crystallogr., Sect. B: Struct. Sci., 1985, B41, 131-135, Anhydrous Oxalic Acid e.g. CCDC Refcodes OXALAC03 and OXALAC04; J. L. Derissen and P. H. Smit, Acta Crystallogr., Sect. B: Struct. Crystallogr. Cryst. Chem., 1974, B30, 2240-2242.

7 C. O. Agbada, Ph.D. Thesis, University of Bradford, 1991.

8 T. Gelbrich and M. B. Hursthouse, CrystEng Comm, 2005, 7, 324-336.

9 K. K. Rajendra, L. Devalina and D. J. Grant, Int. J. Pharm., 1992, 82, $117-127$.

10 M. O. Okoth, Ph.D Thesis, University of Strathclyde, Scotland, UK, (2002).

11 M. P. Sudhakaran and T. D. Radhkrishnan Nair, Asian J. Chem., 1999, 11(1), 270-272; G. T. Guarini and L. Dei, Thermochim. Acta, 1995, 269/270, 79-88.

12 R. Surana, A. Pyne and R. Suryanarayanan, AAPS PharmSciTech, 2003, 4, 1-10.

13 M. L. Smith and B. Topley, Proc. R. Soc. Lond., 1931, A134, 224-245.

14 J. L. Torgesen and J. Strassburger, Science, 1964, 146, 53-55.

15 M. D. King and T. M. Korter, J. Phys. Chem. A, 2010, 114, 7127-7138. 